Precise Stellar Radial Velocities

ASP Conference Series, Vol. 185, 1999

J. B. Hearnshaw and C. D. Scarfe, eds.

\title{
Discovering new spectroscopic components in visual multiple stars
}

\author{
A.A. Tokovinin \\ Sternberg State Astronomical Institute, Universitetsky prosp., 13, \\ 119899 Moscow, Russia
}

\begin{abstract}
A radial velocity survey of the components of visual multiple stars has been done since 1994 with the correlation radial velocity spectrometer. About 50 spectroscopic sub-systems have been discovered, and a summary of the 30 published orbits is given. Difficulties of measuring the radial velocities of multiple stars are discussed and illustrated by 2 examples. New discoveries will lead to a better understanding of the formation of multiple stellar systems. Close and wide sub-systems can not be considered as a result of independent random combination, but are related by common origin. Almost all close binaries with periods less than 6 days possibly have more distant components which assisted in the formation of close pairs by taking away their angular momentum. On the other hand, among the distant visual tertiary companions the frequency of sub-systems with periods under 100 days is about 3 times higher than for field $\mathrm{G}$ dwarfs.
\end{abstract}

\section{Correlation radial velocities of multiple stars: progress report}

Our current knowledge of multiple star statistics (by multiple we mean here systems of multiplicity 3 or higher) is very poor. Most of these objects were discovered by chance, so the selection effects are very severe. The catalogue of physical multiple stars (Tokovinin 1997a, hereafter MSC) is a first attempt to summarize the available historical data. Its published version contains 612 physical multiple systems; about the same number of uncertain or optical systems is also kept in the private database for further study. For most of the systems even the exact multiplicity is not known, because additional components may be discovered in the future.

During the MSC compilation it became apparent that for many objects or faint components the radial velocities have never been measured. This is why a systematic program of radial velocity observations of multiple stars was started in 1994. We use the correlation radial velocity spectrometer (Tokovinin 1987), which means that only components of the spectral type later than F5 and brighter than $11^{m}$ are accessible. The instrument has a light-scrambling slit, which improves the precision and helps in observations of partially resolved visual components. The aim of our survey is twofold: i) to check for physical relation of faint visual components, and ii) to discover new spectroscopic subsystems. Here we discuss the results obtained so far. 
Table 1. Summary of new discoveries

\begin{tabular}{|c|c|c|c|c|c|}
\hline $\mathrm{HD} / \mathrm{BD}$ & Other & Type & $P$, days & $e$ & Reference \\
\hline $895 \mathrm{C}$ & ADS $161 \mathrm{C}$ & SB2 & 6.026 & 0.025 & PAZh 243431998 \\
\hline 2333 & $\mathrm{ADS} 363 \mathrm{~A}$ & SB1 & 6.390 & 0 & PAZh 214451995 \\
\hline$+49435 \mathrm{C}$ & ADS $1315 \mathrm{C}$ & SB1 & 2.222 & 0 & A\&AS 1218461997 \\
\hline $10308 \mathrm{~B}$ & ADS $1326 \mathrm{~B}$ & SB1 & 1.435 & 0 & PAZh 243431998 \\
\hline 15144 & ADS $1849 \mathrm{~A}$ & SB1 & 2.999 & 0.03 & A\&AS 1218461997 \\
\hline $22091 \mathrm{C}$ & ADS $2616 \mathrm{C}$ & $\mathrm{SB} 2$ & 3.748 & 0 & PAZh 243431998 \\
\hline $27638 \mathrm{~B}$ & $\mathrm{ADS} 3161 \mathrm{~B}$ & SB2 & 17.60 & 0.303 & new, triple \\
\hline 28363 & ADS $3248 \mathrm{~B}$ & SB1 & 21.25 & 0.268 & PAZh 214451995 \\
\hline 32093 & ADS $3608 \mathrm{C}$ & $\mathrm{SB} 2$ & 186.28 & 0.343 & A\&AS 1218461997 \\
\hline $33959 \mathrm{C}$ & ADS $3824 \mathrm{C}$ & SB1 & 2.993 & 0 & A\&AS 1218461997 \\
\hline 35317 & ADS $3991 \mathrm{~A}$ & SB2 & 22.58 & 0.610 & A\&AS 1218461997 \\
\hline 48767 & ADS $5436 \mathrm{~B}$ & SB1 & 4.258 & 0.053 & newa \\
\hline 67064 & $\mathrm{ADS} 6646 \mathrm{~A}$ & SB1 & 4.884 & 0 & A\&AS 1218461997 \\
\hline $82780 \mathrm{~B}$ & ADS $7438 \mathrm{~B}$ & SB1 & 28.23 & 0.44 & PAZh 243431998 \\
\hline 92855 & HR $4191 \mathrm{~B}$ & SB1 & 5.611 & 0.324 & PAZh 208261994 \\
\hline 100831 & ADS $8236 \mathrm{~B}$ & SB2 & 1690.1 & 0.647 & new ${ }^{a}$ \\
\hline+352436 & ADS $8861 \mathrm{~A}$ & $\mathrm{SB} 2$ & 200.26 & 0.531 & A\&AS 1218461997 \\
\hline 124640 & ADS $9167 \mathrm{~A}$ & SB1 & 1047.8 & 0.754 & PAZh 247721998 \\
\hline 139691 & ADS $9731 \mathrm{~A}$ & SB2 & 3.273 & 0 & PAZh 249181998 \\
\hline 139691 & ADS $9731 \mathrm{D}$ & SB1 & 14.28 & 0.31 & PAZh 249181998 \\
\hline 148086 & ADS $10044 \mathrm{~A}$ & SB1 & 21.59 & 0.03 & new ${ }^{a}$ \\
\hline+043562 & Kui $84 \mathrm{~B}$ & SB2 & 34.50 & 0.386 & PAZh 203681994 \\
\hline 166866 & ADS $11061 \mathrm{~A}$ & SB2 & 1247.2 & 0.9754 & PAZh 212861995 \\
\hline 238865 & ADS $11336 \mathrm{C}$ & SB1 & 2.710 & 0 & PAZh 212831995 \\
\hline 178091 & ADS $12040 \mathrm{~A}$ & SB2 & 27.25 & 0.338 & PAZh 204071994 \\
\hline 178911 & ADS $12101 \mathrm{~A}$ & SB1 & 1288.8 & 0.615 & new \\
\hline $191104 \mathrm{~B}$ & ADS $13403 \mathrm{~B}$ & SB1 & 23.84 & 0.123 & PAZh 243431998 \\
\hline 193797 & Cou $327 \mathrm{~A}$ & SB1 & 70.53 & 0.463 & PAZh 205131994 \\
\hline 214511 & $\operatorname{ADS} 16111 \mathrm{~A}$ & SB1 & 4.570 & 0.029 & PAZh 243431998 \\
\hline 216963 & Cou $542 \mathrm{~A}$ & SB1 & 118.48 & 0.180 & PAZh 205131994 \\
\hline
\end{tabular}

A\&AS $=$ Astronomy and Astrophysics Supplement Series.

PAZh = Pis'ma v Astronomicheskii Zhurnal (English translation: Astronomy Letters).

\footnotetext{
${ }^{a}$ Published since the Colloquium in A\&AS 1363731999
}

Of the 480 components in our program about 390 have been observed. A total of 2408 observations have been obtained in 1994-1998 with the 1-m telescope in Crimea and 0.7-m telescope in Moscow. In a parallel effort, my colleague M. Smekhov is measuring the radial velocities of visual binaries with known orbits, in order to find closer sub-systems (333 objects, 1663 measurements). This second program can be considered as a continuation of the work of Petrie and Batten (1965) with modernobserving techniques.

Table 1 contains the summary of the published spectroscopic orbits which resulted from these 2 projects. Also included are our previous discoveries of spectroscopic components in multiple stars and new orbits ready for publication. To these 30 orbits will be added another 7 objects with preliminary orbits and 15 objects with variable radial velocities and yet unknown periods. Thus the total score of new discoveries is actually around 50 .

These new discoveries improve the knowledge of stellar multiplicity statistics. For multiple stars the number of orbital parameters is larger than for binary stars, and there is a hope to find new clues to star formation from additional 
data like mutual orbit orientation, period ratio, correlation between mass ratios, etc. Each discovery fills the parameter space with a new point, and there is a chance to find special objects with extreme parameters. For example, spectroscopic orbits with the highest eccentricities are found in multiple stars (e.g. HD 166866 with $e=0.9754$ ), or high-multiplicity systems can be discovered like HD 139691 (the highest multiplicity actually known is 6 , and only 4 certain hierarchical sextuples are listed in MSC).

\section{Problems with multiple star velocities}

Radial velocity observations of multiple stars are specific because the separation between visual components defines what is actually measured spectroscopically. The easiest case is when the components are fully resolved (separation $>10^{\prime \prime}$ ). In this instance a single observation of a large difference between the components' velocities may indicate that one of them is a spectroscopic binary - quite a favorable situation for discovery as compared to single stars!
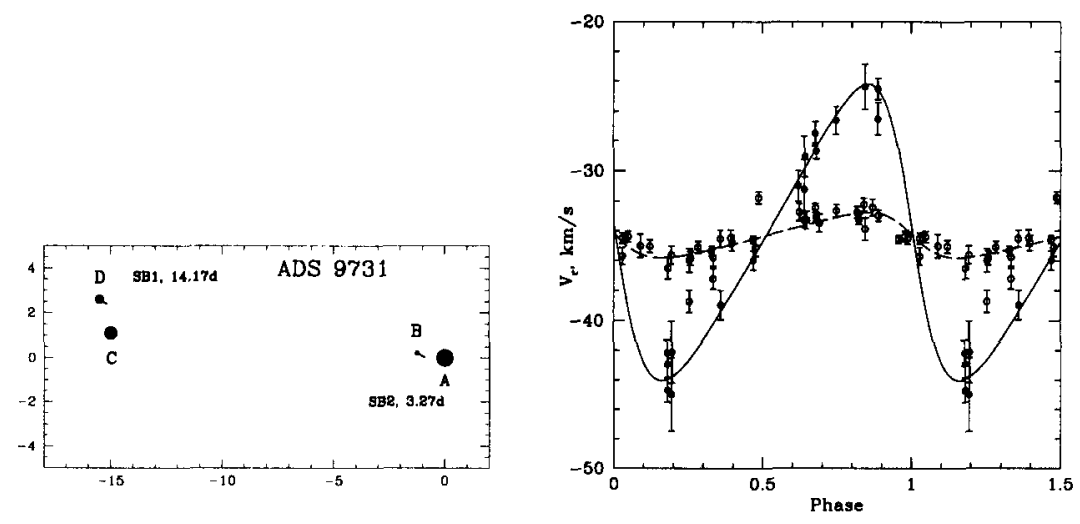

Figure 1. The sextuple system ADS 9731 = HD 139691. Left: the configuration of the 4 visual components on the sky is shown (north up, east to the left, scale in arcseconds). New spectroscopic subsystems are marked. Right: the radial velocity curve of ADS $9731 \mathrm{D}$ (filled circles and full line) and of the combined light of CD (empty circles and dashed line) showing the effect of components' mixing on the slit.

When the visual components have a separation less than $1^{\prime \prime}$ they are usually fully unresolved. If the components' velocities are substantially different, they can be measured as in an ordinary double-lined binary. However, the problem of cross-identification between the spectroscopic and visual components usually exists. Let us suppose that the visual primary is itself a close double-lined binary; then the lines of the visual secondary may have the greatest contrast in the combined spectrum, and then the close sub-system may be erroneously attributed to the visual secondary. 
Most difficult is the case of partially resolved components (separation from $1^{\prime \prime}$ to $\left.10^{\prime \prime}\right)$. Light of both components is mixed in a spectrograph slit or fiber in a proportion which depends on seeing and telescope pointing. This variable and uncontrolled mixing introduces additional scatter in the measured velocities which can not be avoided by using high-precision velocity techniques.

For example, the sextuple system HD 139691 = ADS 9731 contains 4 visual components (Fig. 1a). The primary is a double-lined binary with a short period and large velocity amplitude. Here the additional contribution of the faint visual component $B$ (which is hardly resolved even under the best seeing) is not troublesome. The radial velocity of the mixed light of $\mathrm{CD}$ is slightly variable, and the period of 14 days can be determined. The partial resolution of this $1.6^{\prime \prime}$ pair under good seeing conditions reveals that the spectroscopic sub-system is associated with D (Fig. 1b). The shape of the radial velocity curve of D is not similar to the shape of the CD curve, because at maximum and minimum the combined correlation profile becomes wide and distorted. Also, the large scatter of the "combined" CD velocities is apparent, due to the varying proportion of the components' mixing.
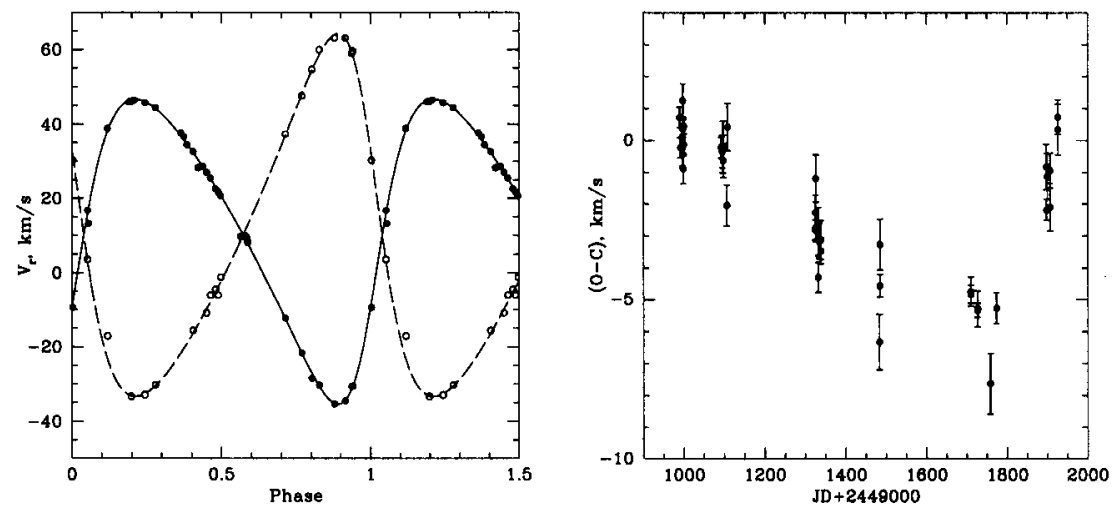

Figure 2. The visual secondary ADS 3161B $=$ HR 1369B was found to be a double-lined spectroscopic binary with a 17.6 day period (left). However, the residuals to the orbit display a systematic behavior revealing the presence of a massive but invisible tertiary with a period of few years (right).

When there are no short-period subsystems but the velocities of the partially resolved binary components are different due to their slow motion in a visual orbit, the "combined" velocity would still show a larger than usual scatter due to the variable mixture of components. A false discovery of a spectroscopic binary may result. The sign of the radial velocity difference can be determined explicitly by measuring the velocity with opposite image offsets on the slit (Smekhov \& Tokovinin 1993).

It is clear that radial velocity observations of partially resolved multiples will greatly benefit from the increased spatial resolution offered by adaptive optics (AO). A step in this direction has been taken with the construction of 
the GRAF imaging spectrograph for the ADONIS AO system (le Coarer et al., 1996).

Despite the problems outlined above, the power of radial velocities in discovering new multiple star components with periods up to several years can not be overestimated. Measurements with good precision are essential. For example, the secondary in a young visual binary ADS $3161=\mathrm{HR} 1369$ was found to be a double-lined spectroscopic binary with a 17.6 day period (Fig. 2a). But on closer examination, it turns out that its systemic velocity changes in a regular way, revealing the presence of a rather massive (but invisible) tertiary with a period of few years (Fig. 2b). If the measurement precision were lower, this additional component would never have been detected.

\section{What can be learned about multiple star formation?}

The birth of close binary stars presents a formidable problem to theory, because the orbit size is much less than the initial size of the protostars. We have to assume that orbital period decreased after the components were formed. The frequent occurrence of close binaries in systems of higher multiplicity suggests that angular momentum and energy could be removed from the close pair through its interaction with the tertiary. Recently this assertion was substantiated by Kiseleva \& Eggleton (1998) and Beust et al. (1997).

The period distribution of the 30 orbits listed in Table 1 is far from uniform. The largest concentration (33\%) is in the range from 2.7 to 6.4 days, no orbits are found in the next period bin (6.4 to 14 days), another $25 \%$ have periods from 17 to 60 days. A larger sample of the spectroscopic binaries from MSC also shows overabundance of subsystems with periods 2-5 days in comparison with the 5-15 day period range.

The formation of subsystems with periods from 2 to 6 days from initially wider binaries can be viewed as a result of angular momentum transfer to the tertiary, bringing two protostellar components into close proximity either in a highly eccentric orbit or during the interplay in a young non-hierarchical triple. Then dissipative forces due to tidal friction and mass transfer take away the orbital energy, decreasing the semimajor axis to its present value and circularizing the orbit. The presence of the tertiary, however, will still "pump" up the eccentricity to small but measurable values in these close binaries which would otherwise have circular orbits. Sometimes a non-zero eccentricity of a late-type close binary can be considered as a sign of its higher multiplicity (Mazeh 1990). The orbit of HD 92855 with $P=5.6 d$ and $e=0.32$ (Table 1) indicates that a close tertiary remains to be discovered.

The idea of the formation of close binaries in multiple systems finds some support when the eighth catalogue of spectroscopic binary star orbits (Batten, Fletcher, \& MacCarthy 1989) is compared to the MSC. It turns out that the period distribution of the spectroscopic binaries belonging to multiple systems is significantly different from the distribution of binaries which are not known to have additional companions (Tokovinin 1997b): the former have a much larger fraction of close subsystems. We believe that this difference is real, because discoveries of spectroscopic and visual sub-systems were independent. It is note- 
worthy that all 5 close (1-10 days) binaries in the Duquennoy \& Mayor (1991) sample are in fact members of higher multiplicity systems.

Now, if a genetic relation between wide and close binaries exists, we can check it by observing the faint distant physical components to the known visual or spectroscopic binaries. From our multiple star survey we selected 35 such components which did not have any previous radial velocity data and had been observed by us with a time span of at least 1 year. In this subsample, 5 objects have periods from 1 to 10 days, 3 from 10 to 100 days, and 6 have variable velocities with periods probably longer than 100 days. So at least $22 \%$ of the components observed (of spectral types from F5V to K0V) are found to be spectroscopic binaries with periods less than 100 days. From the period distribution of G-dwarf binaries given by Duquennoy \& Mayor (1991) it can be inferred that the expected fraction of such binaries is $7.3 \%$. So there is a factor of 3 excess of spectroscopic binaries, in comparison with the field dwarfs. On the other hand, this fraction of close binaries may be typical for cluster stars.

\section{Conclusions}

Multiple stars offer a rich and promising research subject. Their study will greatly benefit from the combination of different observing techniques (radial velocities, interferometry, astrometry). As a result, a volume-limited sample of multiple stars will be defined to study the statistics of stellar multiplicity.

Radial velocity observations of multiple stars should be extended to hot components. A medium resolution fiber-fed echelle spectrograph has been recently constructed at Sternberg Astronomical Institute for this purpose.

\section{References}

Batten, A.H., Fletcher, J.M., \& MacCarthy, D.G. 1989, Publ. Dom. Astrophys. Obs., 17

Beust, H., Corporon, P., Siess, L., \& Forestini, M. 1997, A\&A, 320, 478

le Coarer, E., Chalabaev, A., Rabou, D., Magnard, Y., \& Petmezakis, P. 1996, GRAF instrument description. LAORG Tech. Rep. No. 6 (http://wwwlaorg.obs.ujf-grenoble.fr/hra/graf/)

Duquennoy, A., \& Mayor, M. 1991, A\&A, 248, 485

Kiseleva, L.G., \& Eggleton, P.P. 1998, in Cool Stars, Stellar Systems and the Sun (ASP Conf. Ser., 154), R.A. Donahue \& J.A. Bookbinder, San Francisco, Astron. Soc. Pacific, 2118

Mazeh, T. 1990, AJ, 103, 1357

Petrie, R.M, \& Batten, A.H. 1965, Trans. IAU, 12B, 476

Smekhov, M.G., \& Tokovinin, A.A. 1993, PAZh, 19, 193

Tokovinin, A.A. 1987, AZh, 64, 196

Tokovinin, A.A. 1997a, A\&AS, 124, 75

Tokovinin, A.A. 1997b, PAZh, 23, 834 


\section{Discussion}

Mazeh: I want to congratulate you on this fine work. Two questions: (1) can you comment on teh frequency of multiple systems? (2) For a short-period eccentric binary, why can the distant component not induce the eccentricity?

Tokovinin: (1) I avoid any estimate of multiple star frequency, because the selection effects are too bad. (2) The binary HD 92855 , with a short period and non-zero eccentricity, has a visual companion too distant, so that its perturbations are too weak to be accumulated, as is the case for the 16 Cyg B planet. So there must be a closer and yet undiscovered companion to this star.

Rucinski: The staff of CFHT are now conducting a survey of high-velocity stars for the presence of third components, using the adaptive optics system. A separate program, a survey of contact binaries that I am conducting, to confirm a subjective impression that they always have third components, indeed seems to confirm their high frequency of incidence. Both programs are in progress and will give results in a year or two.

Latham: As chairman, I note that there is a short-period binary with a 4-day period and eccentricity 0.3 in M67. Thus its age is known ... but it is a blue straggler! 